La Revue

des Droits

de l'Homme

\section{La Revue des droits de l'homme}

Revue du Centre de recherches et d'études sur les droits fondamentaux

$1 \mid 2012$

Revue des droits de l'homme $-\mathrm{N}^{\circ} 1$

\title{
Chapitre 3. La justiciabilité de l'obligation alimentaire entre parents et alliés
}

Quels enseignements pour la justiciabilité des droits sociaux?

\section{Marc Pichard}

\section{OpenEdition}

\section{Journals}

Édition électronique

URL : https://journals.openedition.org/revdh/155

DOI : $10.4000 /$ revdh. 155

ISSN : 2264-119X

Éditeur

Centre de recherches et d'études sur les droits fondamentaux

\section{Édition imprimée}

Date de publication : 1 juin 2012

Pagination : 426-439

Référence électronique

Marc Pichard, «Chapitre 3. La justiciabilité de l'obligation alimentaire entre parents et alliés », La Revue des droits de l'homme [En ligne], 1 | 2012, mis en ligne le 30 juin 2012, consulté le 21 septembre 2021. URL : http://journals.openedition.org/revdh/155; DOI : https://doi.org/10.4000/revdh.155 


\section{CHAPITRE 3}

\section{LA JUSTICIABILITE DE L'OBLIGATION ALIMENTAIRE ENTRE PARENTS ET ALLIES : QUELS ENSEIGNEMENTS POUR LA JUSTICIABILITE DES DROITS SOCIAUX ?}

\section{MARC PICHARD}

«Les enfants doivent des aliments à leurs père et mère ou autres ascendants qui sont dans le besoin " ${ }^{1220}$; " les gendres et les belles-filles doivent également, et dans les mêmes circonstances, des aliments à leur beau-père et belle-mère "1221 ; "les obligations résultant de ces dispositions sont réciproques " ${ }^{1222}$. A lire le Code civil, les secours en cas de besoin sont à attendre non pas de la collectivité mais de la famille ou, plus précisément, des parents et alliés ${ }^{1223}$. Et l'impression sort renforcée de la consultation d'autres sources. L'article L. 132-6 du Code de l'action sociale et des familles ne dispose-t-il pas que "les personnes tenues à l'obligation alimentaire instituée par les articles 205 et suivants du code civil sont, à l'occasion de toute demande d'aide sociale, invitées à indiquer l'aide qu'elles peuvent allouer aux postulants et à apporter, le cas échéant, la preuve de leur impossibilité de couvrir la totalité des frais ", illustrant de la sorte le principe traditionnel de subsidiarité de l'aide de la collectivité par rapport à l'aide familiale ?1224

Or, au premier abord, cette origine en principe familiale des aliments semble éloigner la question du thème des droits sociaux ${ }^{1225}$. On a en effet du mal à concevoir que l'obligation alimentaire entre parents et alliés puisse être un vecteur de la justice sociale. Parfois, l'effet pourrait même être contraire. Car il n'est certes pas rare que les parents et alliés du pauvre soient pauvres, les parents et alliés du riche, riches. Or la mesure de l'obligation est le rapport

\footnotetext{
1220 C. civ., art. 205.

1221 C. civ., art. 206, qui précise: "mais cette obligation cesse lorsque celui des époux qui produisait l'affinité et les enfants issus de son union avec l'autre époux sont décédés ».

1222 C. civ., art. 207, al. $1^{\mathrm{er}}$.

${ }^{1223}$ Le droit civil raisonne bien plus en termes de liens interindividuels que de droit contre un groupe, de sorte qu'il est assez discutable de parler d'obligation alimentaire familiale: v. Dominique EVERAERT-DUMONT, «Le paradoxe des obligations alimentaires ou comment concilier principe de solidarité et obligation personnelle ", RDSS, 2008, p. 538 et s., spéc. p. 541 et s.

${ }^{1224}$ Sur la question v., outre les références citées supra, pp. 377-378, Jean HAUSER, « La famille récupérée ", in Le droit privé français à la fin du XXe siècle, Etudes offertes à Pierre Catala, Litec, 2001, p. 327 et s.

1225 Supra, p. 5.
} 
entre les besoins du créancier et les ressources du débiteur ${ }^{1226}$. À celui qui a besoin de beaucoup, car son train de vie est assez élevé, et dont les débiteurs ont beaucoup, il sera beaucoup fourni. À celui qui, pour avoir toujours été pauvre, a besoin de peu, et dont les parents et alliés n'ont guère, il sera peu promis ${ }^{1227}$. La fixation judiciaire de l'obligation alimentaire prend parfois des allures de droit de classe.

L'objection ne semble toutefois pas dirimante. Car, incontestablement, fait partie du corpus de l'étude, le droit à des moyens convenables d'existence ${ }^{1228}$ - et la mise en œuvre de l'obligation alimentaire peut permettre de les obtenir. Or ce serait restreindre arbitrairement la question des débiteurs de ce droit social que de poser, $a b$ initio, qu'il ne pourrait contraindre que la collectivité. Personne ne conteste que, par la reconnaissance d'un effet horizontal aux stipulations de la Convention européenne des droits de l'Homme, la Cour de Strasbourg a amélioré l'effectivité des droits, en particulier civils et politiques. Les États, pour respecter leurs engagements internationaux, doivent donc protéger les droits consacrés y compris des atteintes des particuliers. Tout pareillement, en imposant à des personnes privées la charge de la satisfaction d'un droit social, et dès lors que son intervention ne se limite pas à cela, un État œuvre à sa garantie, puisqu'il améliore la situation de son titulaire. À cet égard, la consécration du droit à l'habitat puis du droit au logement dans la législation française est éclairante : ce sont des lois relatives aux baux d'habitation qui, les premières, les ont portés ${ }^{1229}$, et dont l'objet est d'accroître la contrainte pesant sur le propriétaire - irréfragablement présumé en situation de force, mais qui peut ne pas l'être - au bénéfice du locataire - irréfragablement présumé dans une situation moins confortable. Que cette législation participe à la protection du droit au logement, comme l'affirment les textes, est plus que probable: l'instauration de charges et contraintes sur les personnes privées est un des modes de garantie d'un droit social. Or, en matière d'obligation alimentaire, contrainte il y a. L'obligation alimentaire n'est pas abandonnée par l'État aux relations familiales privées, elle n'est pas une obligation naturelle ${ }^{1230}$ : son exécution peut être imposée en justice.

1226 C. Cass., C. civ., art. 208, al. $1^{\text {er }}$ : "Les aliments ne sont accordés que dans la proportion du besoin de celui qui les réclame, et de la fortune de celui qui les doit ».

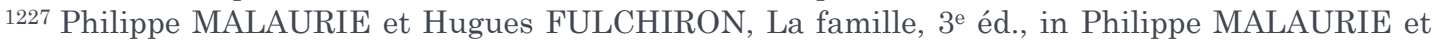
Laurent AYNES, Droit civil, Defrénois, 2009, n 1748 : « Le montant des droits alimentaires [...] est apprécié en tenant compte du genre de vie et de la condition sociale des intéressés; dans certains cas, la pension alimentaire est donc inférieure au salaire minimum, dans d'autres, elle est très élevée". Le besoin est irréductible à la pauvreté : Diane ROMAN, Le droit public face à la pauvreté, LGDJ, coll. "Bibliothèque de droit public", 2002, n 146 et s. - qui, en particulier, souligne la subjectivité de l'appréciation des besoins du créancier dans le cadre de la détermination du quantum de l'obligation alimentaire.

1228 Supra, p.5.

1229 V., toujours en vigueur, la loi $\mathrm{n}^{\circ} 89-462$ du 6 juillet 1989 tendant à améliorer les rapports locatifs et portant modification de la loi n 86-1290 du 23 décembre 1986, dite Mermaz (JO 8 juillet 1989 , p. 8541), et son article $1^{\text {er }}$ : « Le droit au logement est un droit fondamental ; il s'exerce dans le cadre des lois qui le régissent".

1230 Sur la définition de l'obligation naturelle, v. supra, p. 379, note 17. 
Et le souci de voir cette obligation alimentaire respectée par ses débiteurs personnes privées a donné lieu à des interventions très spécifiques. Non seulement le législateur a conféré à la créance alimentaire la protection du juge à laquelle tous les droits reconnus par la législation civile peuvent prétendre, mais l'obligation alimentaire bénéficie d'une justiciabilité accrue car sa satisfaction se révèle entourée de garanties procédurales propres : "le but spécifique de l'obligation alimentaire, qui est d'assurer la subsistance du créancier, ainsi que son fondement particulier, qui est le devoir de charité et de solidarité familiales, expliquent qu'elle soit soumise à un régime juridique très spécial qui, sur de nombreux points, se distingue de celui qui vaut pour les obligations ordinaires "1231. Sa réalisation en justice est facilitée par diverses solutions et dispositions qui ne s'appliquent qu'à elle. De fait, cette justiciabilité accrue peut s'expliquer par les difficultés spécifiques de recouvrement des créances en question : elle est probablement le produit d'une ineffectivité ellemême non négligeable ${ }^{1232}$. Son étude est d'autant plus importante.

Confrontés à une forme d'ineffectivité de la créance alimentaire, mode de satisfaction du droit à des moyens convenables d'existence, les pouvoirs publics ont posé des règles qui tendent à favoriser sa justiciabilité, c'est-à-dire l'exercice d'une contrainte contre les parents et alliés. Se pose alors la question de savoir si, de ce régime spécifique, des enseignements à portée plus générale pourraient être tirés : ces modes originaux de garantie de la créance alimentaire peuvent-ils nourrir la réflexion sur la justiciabilité des droits sociaux? En matière d'obligation alimentaire entre parents et alliés, deux voies d'une justiciabilité meilleure sont empruntées : le recours au juge est favorisé ; le recouvrement de la créance est facilité.

\section{Le recours au juge favorisé}

L'obligation alimentaire ne peut être comprise qu'au regard de l'état de besoin, qui en conditionne l'existence-même. Or cet état est, justement, de nature à compliquer l'accès au juge comme à pousser à renoncer à la garantie que constitue le recours à celui-ci. Une meilleure justiciabilité du droit passe précisément par la protection contre ces deux écueils.

\section{A. Une accessibilité renforcée}

Rationae materiae, le juge de l'obligation alimentaire est un juge facilement identifiable : c'est le juge aux affaires familiales, juge du tribunal de

1231 J. Cl. Civil, art. 205 à 211, fasc. 20 : Aliments - Obligation alimentaire - Caractères, 2002, par Laurent LEVENEUR, $\mathrm{n}^{\circ} 1$. C'est une "créance privée [...] d'une nature particulière " (Jean HAUSER et Danièle HUET-WEILLER, Traité de droit civil, dir. Jacques GHESTIN, La famille, Fondation et vie de la famille, $2^{\mathrm{e}}$ éd., LGDJ, 1993, n 1350 , p. 903).

1232 En ce sens, v. Jean CARBONNIER, Droit civil, t. 2, La famille, l'enfant, le couple, 21e éd., PUF, coll. «Thémis ", 2002, p. 60 : « La législation favorable aux créanciers [...] est plutôt le signe d'un mauvais fonctionnement de l'institution "; Jean HAUSER et Danièle HUET-WEILLER, précit., n 1342 , p. 900. 
grande instance ${ }^{1233}$. Le caractère très général de sa compétence favorise probablement la justiciabilité du droit, dans la mesure où il évite que des incertitudes en la matière freinent le recours au juge. En outre, alors que, en principe, la représentation est obligatoire devant le tribunal de grande instance et ses diverses formations, l'article 1139 du Code de procédure civile dispense du ministère d'avocat certaines procédures relevant de la compétence du juge aux affaires familiales, parmi lesquelles le contentieux de l'obligation alimentaire. Une entrave à la saisine du juge par les personnes les plus modestes est de la sorte levée ${ }^{1234}$.

Ce souci d'un moindre coût à la charge d'un demandeur dans le besoin anime aussi les règles, dérogatoires, relatives à la compétence rationae loci : le juge de l'obligation alimentaire - même s'il n'est pas la juridiction de proximité ${ }^{1235}$ - est un juge proche. En effet, alors que le juge territorialement compétent est en principe celui du domicile du défendeur, ce qui peut conduire à devoir saisir une juridiction très éloignée - avec tous les surcoûts que cela comporte (même pour une personne bénéficiaire de l'aide juridictionnelle) -, en vertu de l'article $46 \mathrm{du}$ Code de procédure civile, le juge du domicile du défendeur est également, en matière alimentaire, compétent ${ }^{1236}$, et une telle option est aussi ouverte, concernant un litige transfrontalier au sein de l'Union européenne, par le règlement du 18 décembre 2008, relatif à la compétence, la loi applicable, la reconnaissance et l'exécution des décisions et la coopération en matière d'obligations alimentaires ${ }^{1237}$. Ainsi des frais sont-ils épargnés à celui qui, par hypothèse, prétend être - et est probablement - dans le besoin ${ }^{1238}$. La conscience de l'importance de rattacher le plus possible la créance alimentaire au lieu de vie du créancier s'exprime également à l'article 1247 du Code civil : alors que, en principe, "le payement doit être fait au domicile du débiteur", c'est-à-dire que la créance est quérable, « les aliments alloués en justice doivent être versés, sauf décision contraire du juge, au domicile ou à la résidence de celui qui doit les recevoir » - la créance alimentaire est portable ${ }^{1239}$. Le droit civil tend

${ }^{1233}$ C. org. jud., art. L. 213-3 ; adde c. santé pub., art. L. 6145-11 : « Les établissements publics de santé peuvent toujours exercer leurs recours, s'il y a lieu, contre les hospitalisés, contre leurs débiteurs et contre les personnes désignées par les articles 205, 206, 207 et 212 du code civil. Ces recours relèvent de la compétence du juge aux affaires familiales ».

${ }^{1234}$ Certes, l'admission à l'aide juridictionnelle de ces mêmes personnes leur permettrait d'obtenir l'assistance d'un avocat. Mais les délais de traitement des dossiers sont souvent longs - et la procédure en elle-même peut parfois rebuter certaines d'entre elles.

${ }_{1235} \mathrm{Au}$ sens de l'art. L. 231-3 du Code de l'organisation judiciaire.

1236 C. proc. civ., art. 46 : "Le demandeur peut saisir à son choix, outre la juridiction du lieu où demeure le défendeur : [...] en matière d'aliments ou de contribution aux charges du mariage, la juridiction du lieu où demeure le créancier "; la solution est rappelée à l'article 1070, al. 3.

1237 Règlement $(\mathrm{CE}) \mathrm{n}^{\circ}$ 4/2009 du Conseil du 18 décembre 2008, relatif à la compétence, la loi applicable, la reconnaissance et l'exécution des décisions et la coopération en matière d'obligations alimentaires, JO UE 10 janvier 2009, n L 7, art. 3

1238 « Le créancier a une option [...], justifiée par la précarité de son état» : Frédérique GRANET, "Créances alimentaires: solidarité familiale ou solidarité sociale?", in La dépendance des personnes âgées, dir. Francis KESSLER, 2e éd., Sirey, coll. " Droit sanitaire et social », 1997, p. 115 et s., p. 121.

1239 Sur la distinction, v. François TERRE, Philippe SIMLER et Yves LEQUETTE, Droit civil. Les obligations, 10éd., Dalloz, coll. "Précis ", 2009, n 1344, p. 1334-1335. L’enjeu principal est de 
à favoriser la justiciabilité du droit en faisant peser sur le débiteur potentiel ou avéré de la créance la charge de la distance, en particulier de la distance au juge : l'exemple mériterait d'être médité ${ }^{1240}$.

\section{B. Un garant exceptionnel}

Que le juge civil ait à protéger les droits de la personne, surtout si leur siège est à trouver dans le Code civil, n'a rien d'étonnant. Mais la vocation du juge à se prononcer sur l'existence et le quantum de l'obligation alimentaire est tout à fait particulière : son rôle de garant s'avère exceptionnel.

D’une part, le juge est tout spécialement garant de la créance alimentaire parce que celle-ci est indisponible - du moins les sommes à échoir à ce titre ${ }^{1241}$. Cette indisponibilité se traduit par une justiciabilité accrue, au sens où elle favorise l'intervention d'une juridiction.

Débiteurs et créanciers peuvent, en dehors de tout litige, fixer conventionnellement le quantum de l'obligation alimentaire. C'est d'ailleurs bien souvent par accord tacite entre le créancier et le débiteur que l'obligation alimentaire est exécutée. Simplement, quand bien même un tel accord serait susceptible d'être prouvé, car explicité, il n'obligerait guère les parties : sa force obligatoire est quasi-nulle ${ }^{1242}$. En effet, il est toujours loisible au créancier comme d'ailleurs au débiteur - de saisir le juge pour qu'il révise le montant de la pension alimentaire à verser, nonobstant tout accord antérieur entre les parties $^{1243}$.

savoir quelles sont les formalités préalables à une poursuite. Lorsque la dette est quérable, en pratique, "cette règle signifie aujourd'hui que le créancier ne peut pas valablement poursuivre le débiteur ou le mettre en demeure s'il ne lui a pas demandé le paiement, par exemple par courrier adressé à son domicile" (p. 1334). Si la créance est portable, "la mise en demeure et des poursuites sont possibles dès l'arrivée de l'échéance, sans que le créancier ait à faire preuve d'une demande préalable de paiement» (p. 1335).

1240 Cette volonté de favoriser la proximité du juge en présence d'un demandeur en situation économique présumée fragile a conduit à admettre récemment une option assez proche en matière de droit de la consommation. Depuis l'entrée en vigueur de la loi n² 2009-526 du 12 mai 2009 de simplification et de clarification du droit et d'allègement des procédures, en vertu de l'article L. 141-5 du Code de la consommation, "le consommateur peut saisir à son choix, outre l'une des juridictions territorialement compétentes en vertu du code de procédure civile, la juridiction du lieu où il demeurait au moment de la conclusion du contrat ou de la survenance du fait dommageable». Sur cette nouvelle disposition, v. Dominique FENOUILLET, RDC 2009.1438; Stéphane PIEDELIEVRE, Gaz. Pal. 2009.1732 ; Loïs RASCHEL, Procédures juill. 2009, n 230, p. 17 ; Elise POILLOT, D. 2010.790 , p. 797.

1241 Sur l'indisponibilité de la créance alimentaire, v. J. Cl. Civil, art. 205 à 211, fasc. 20, précit., par Laurent LEVENEUR, $\mathrm{n}^{\circ} 28$ et s., et les réf. citées.

1242 Comp. C. civ., art. 1134, al. $1^{\text {er }}$ : «Les conventions légalement formées tiennent lieu de loi à ceux qui les ont faites".

1243 V. Jean HAUSER, obs. sous Cass. $1^{\text {re }}$ civ. 16 juin 1993, RTD civ. 1993.816 : l'« obligation alimentaire peut faire l'objet d'une convention entre créanciers et débiteurs, étant acquis que cette convention serait toujours susceptible de révision". Adde J. Cl. Civil, art. 205 à 211, fasc. 20, précit., par Laurent LEVENEUR, n 29 : «Le créancier ne saurait convenir avec son débiteur que sa pension sera immuablement fixée à un montant inférieur à celui qu'elle devrait normalement avoir, et qu'elle n'augmentera pas". C'est qu'en effet "le montant des dettes alimentaires est toujours sujet à révision et [...] le créancier ne peut jamais renoncer à ses droits » (Cass. civ. 21 
De même, l'indisponibilité de la créance alimentaire interdit aux parties en litige de compromettre - de soumettre, par une convention, leur litige déjà né à un arbitre ${ }^{1244}$-, comme de transiger - c'est-à-dire de mettre fin à ce litige par un contrat -, la transaction supposant des concessions réciproques, incompatibles avec l'indisponibilité ${ }^{1245}$. Le recours à l'accord de volontés pour qu'il soit mis fin au litige soit directement via par une transaction soit indirectement par saisine d'un arbitre est donc proscrit en matière alimentaire. Le juge étatique jouit d'un monopole.

Ces solutions doivent être rapprochées de l'article L. 132-7 du Code de l'action sociale et des familles. Le texte dispose en effet: "En cas de carence de l'intéressé, le représentant de l'Etat ou le président du conseil général peut demander en son lieu et place à l'autorité judiciaire la fixation de la dette alimentaire et le versement de son montant, selon le cas, à l'Etat ou au département qui le reverse au bénéficiaire, augmenté le cas échéant de la quotepart de l'aide sociale ". En d'autres termes, le droit peut être justiciable contre la volonté du premier intéressé ${ }^{1246}$, alors que, en principe, concernant un droit exclusivement attaché à la personne, l'action oblique n'est pas ouverte au créancier du créancier d'aliments ${ }^{1247}$.

En somme, nonobstant tout accord des parties en sens contraire, ou toute réticence du créancier à se prévaloir de son droit, la créance d'aliments demeure toujours justiciable au sens de susceptible de donner lieu à une décision du jugeet plus précisément du juge étatique. De la sorte s'exprime un certain

janvier 1930 ; Gaz. Pal. 1930, 1, p. 456 : solution adoptée dans un contexte très particulier, mais formule à la portée extrêmement générale; dans le même sens, v. Cass. $2^{\mathrm{e}}$ civ. 18 mars 1954, D. 1954.398).

1244 C. civ., art. 2059 : «Toutes personnes peuvent compromettre sur les droits dont elles ont la libre disposition"; a contrario, l'indisponibilité de la créance alimentaire fait obstacle au compromis. C. civ., art. 2060 : "On ne peut compromettre sur les questions d'état et de capacité des personnes, sur celles relatives au divorce et à la séparation de corps ou sur les contestations intéressant les collectivités publiques et les établissements publics et plus généralement dans toutes les matières qui intéressent l'ordre public"; or il ne fait aucun doute que l'obligation alimentaire relève de cette dernière catégorie.

1245 Sur l'impossibilité de la transaction, v., dans un contexte qui donne à la solution une portée très générale, Cass. civ. 28 février 1949 ; JCP 1949 II 4888, note Paul ESMEIN ; D 1949 J 301, note Georges RIPERT, qui écrit : " le conjoint divorcé qui par insouciance ou générosité a renoncé à sa pension, celui qui a transigé croyant bien faire, pourra se trouver quelques années plus tard privé de tout secours. Il faut le défendre contre lui-même [nous soulignons]. Il est en tout cas de l'intérêt social qu'il ne soit pas un malheureux à la charge de l'Etat [...]. L'arrêt rapporté ne s'arrête pas à l'objection que deux époux divorcés peuvent discuter librement leurs intérêts. Il s'inscrit dans la ligne générale d'un droit protecteur des faibles ». Adde, réitérant la solution, Cass. civ. 23 mai 1949, JCP 1949 II 5202, note Paul ESMEIN.

${ }^{1246}$ La disposition a pu être analysée comme "une aide administrative au recouvrement de la créance familiale» (Frédérique GRANET, art. précit., p. 128). Il ne s'agit toutefois pas stricto sensu d'une aide au recouvrement de la créance au sens où cette aide est alors sollicitée en amont, non pas seulement pour permettre le recouvrement, mais dès avant pour assurer la fixation de la créance. Sur l'aide au recouvrement stricto sensu, v. infra p. 425 et s.

1247 V. en part. J. Cl. Civil, art. 205 à 211, fasc. 20, précit., par Laurent LEVENEUR, n 21 et s., et spéc. $\mathrm{n}^{\circ} 25$ pour une présentation de l'article L. 132-7 du Code de l'action sociale et des familles comme une exception au principe d'exclusion de l'action oblique - exclusion elle-même fondée sur l'article 1166 du Code civil ("Les créanciers peuvent exercer tous les droits et actions de leur débiteur, à l'exception de ceux qui sont exclusivement attachés à la personne »). 
scepticisme à l'égard de l'aptitude du créancier de l'obligation à défendre convenablement ses intérêts, de manière générale mais tout particulièrement dans un cadre conventionnel. Le phénomène n'a rien d'exceptionnel : l'ordre public vient régulièrement protéger, par la technique des renonciations impossibles, le faible - ou présumé tel - contre sa propre volonté, supposée dominée par autrui ${ }^{1248}$. Il n'en est pas moins remarquable dans un contexte de faveur généralisée pour la forme contractuelle - le cas échéant assez abâtardie ${ }^{1249}$. Car envisager la question sociale sous l'angle des droits fondamentaux, c'est-à-dire des droits subjectifs lato sensu, conduit à placer au premier plan la volonté de l'individu titulaire de la prérogative ${ }^{1250}$; le régime de justiciabilité de l'obligation alimentaire rappelle que la libre disponibilité n'est pas toujours le meilleur vecteur du respect des intérêts de la personne, et que concevoir la question en termes d'ordre public est peut-être plus efficace - même si probablement moins satisfaisant pour l'esprit.

D'autre part, si le juge est tout spécialement garant de l'obligation alimentaire, c'est parce que même la fixation judiciaire de la pension ne ferme pas la voie à une nouvelle saisine du juge : les décisions judiciaires sont, en la matière, marquées d'une très forte précarité - car essentiellement provisoires. Il demeure toujours possible de saisir à nouveau le juge pour qu'il procède à une nouvelle évaluation de la pension en fonction de l'évolution des ressources du créancier comme du débiteur: "la décision judiciaire fixant une pension alimentaire ne possède l'autorité de la chose jugée qu'aussi longtemps que demeurent inchangées les circonstances au regard desquelles elle est

\footnotetext{
1248 Sur la méfiance à entretenir à l'égard des " accords de volontés " en présence de parties dans des situations économiquement très différentes, v., en part., Alain SUPIOT, Critique du droit du travail, 2e éd., PUF, coll. "Quadrige ", 2007, p. 116 et s. ; Muriel FABRE-MAGNAN, " Le domaine de l'autonomie personnelle. Indisponibilité du corps humain et justice sociale ", D. 2008.31, spéc. p. 35. Pour une illustration classique, v. Cass. Plén. 4 mars 1983, Bull. civ. n 3 ; D. 1983 J 381, concl. Jean CABANNES : un salarié avait accepté une novation substituant au contrat de travail originaire une "convention de collaboration de type purement libéral»; il demande la requalification de son contrat en contrat de travail, et l'obtient. "Cette activité, exercée sous la dépendance d'un employeur, ne pouvait avoir un caractère libéral, la seule volonté des parties étant impuissante à soustraire [la personne concernée] au statut social qui découlait nécessairement des conditions d'accomplissement de son travail». Les conclusions de l'avocat général Cabannes invitaient à la solution retenue. V. en part., p. 382 : " La volonté des parties a-telle suffi à réaliser une novation? La question est d'autant plus importante que nous débordons aujourd'hui du cadre du droit privé traditionnel et que la détermination des caractères du contrat de travail, dans un secteur se rapprochant du droit public, ou frôlant l'ordre public, non seulement ne peut être laissée à l'initiative des intéressés, mais encore est placée sous votre contrôle : c'est toute la finalité du droit social qui se trouve ici mise en cause!". Pour un parallèle entre ces questions, v. J. Cl. Civil, art. 205 à 211, fasc. 20, précit., par Laurent LEVENEUR, n 34 : « Il en va un peu de "l'ordre public alimentaire" comme de la notion bien connue d'"ordre public social" qui interdit de déroger aux règles légales au détriment du salarié, mais le permet en sa faveur ". 1249 Sur le phénomène de contractualisation, v. en part. Sandrine CHASSAGNARD-PINET et David HIEZ (dir.), Approche critique de la contractualisation, LGDJ, coll. " Droit et société », 2007. 1250 Si l'on veut bien admettre que le droit subjectif appelle la maîtrise de la prérogative par son titulaire, sur quoi v. en part. Jean DABIN, Le droit subjectif, Dalloz, 1952, p. 80 : «Le droit subjectif est essentiellement appartenance-maîtrise, l'appartenance causant et déterminant la maîtrise "; p. 90-91 : le droit subjectif " est faculté d'agir en maître sur la chose qui fait l'objet du droit, naturellement sous réserve des limitations ou des charges résultant soit de la loi, soit de la volonté du titulaire s"auto-limitant" en exécution de sa propre maîtrise. Mais cette limitation même laisse debout, pour le surplus, la libre disposition ".
} 
intervenue ; [...] une demande en révision peut être soumise aux tribunaux, dès lors qu'apparaissent des éléments nouveaux ${ }^{1251}$. Si la perpétuelle réouverture des débats bénéficie tout autant au créancier qu'au débiteur ${ }^{1252}$, elle est indiscutablement le symbole d'une justiciabilité particulière qui n'a que peu d'équivalents en droit civil, sinon en droit de l'assistance éducative ou de l'autorité parentale, où l'intérêt de l'enfant imprime sa marque - d'ordre public à la matière, et confère à toute décision un même caractère provisoire.

Si l'on peut parler d'une justiciabilité spécifique et accrue de l'obligation alimentaire entre parents et alliés, c'est que son régime juridique favorise le recours au juge. Mais accroître la justiciabilité d'un droit c'est aussi s'assurer que, la décision prise, elle pourra être exécutée - que la créance fixée pourra être recouvrée.

\section{Le recouvrement de la créance facilité}

Une fois sa qualité reconnue, le créancier d'aliments bénéficie d'une situation à divers égards exceptionnelle. A rebours de la tendance dominante du droit contemporain, la matière alimentaire révèle en effet une remarquable rigueur à l'égard du débiteur ${ }^{1253}$, dans une situation d'autant plus inconfortable que, pour le recouvrement de la créance, l'administration peut, suivant des voies originales, être mobilisée.

\section{A. La rigueur à l'égard du débiteur}

La faveur pour le débiteur, trait marquant du droit contemporain des obligations, ne trouve certes pas droit de cité en matière alimentaire.

1251 Cass. $1^{\text {re }}$ civ. 16 juin 1993, Bull. civ. I n 216 ; Def. 1993.1360, obs. Jacques MASSIP ; RTD civ. 1993.816, obs. Jean HAUSER. L'arrêt est d'autant plus intéressant que, si le visa de l'article 1351 du Code civil et la lettre de l'attendu de principe invitent à le lire comme relatif à la seule autorité des décisions de justice, sa solution vaut aussi en matière de détermination conventionnelle de la pension alimentaire. En l'espèce, des juges du fond avaient " pris acte » de l'engagement formel du débiteur de laisser, au titre de l'accomplissement de son obligation alimentaire à l'égard de son petit-fils, ce dernier et son ex-bru jouir d'un immeuble dont il était propriétaire, "jusqu'à ce que l'enfant mineur ait quitté ce toit ". Eu égard à l'évolution de la situation des parties, le débiteur demande à pouvoir jouir à nouveau de son bien. Il est débouté par la cour d'appel saisie car il serait « tenu par l'engagement qu'il a pris au cours de l'instance précédente et qui, homologué par la cour d'appel, a l'autorité de la chose jugée "; la cour ajoute que "l'intéressé ne peut révoquer cet engagement, en invoquant un remariage dont il n'avait pas fait une condition résolutoire ". La Cour de cassation censure le raisonnement: "l'engagement pris [...] l'avait été dans le but d'exécuter partiellement en nature son obligation alimentaire; [...] il en résultait que cet engagement pouvait toujours être révisé, soit par un nouvel accord des parties, soit par une décision de justice, en cas de survenance d'éléments nouveaux et sans que puisse être opposée à une convention provisoire la règle de la force obligatoire des contrats ".

1252 L'article 209 du Code civil n'envisage que la révision à la baisse.

${ }^{1253}$ Le terme est employé par M. LEVENEUR (J. Cl. Civil, art. 205 à 211, fasc. 20, précit., n 49 et s.) : " Le caractère d'ordre public de l'obligation alimentaire transparaît encore dans ses sanctions. Il est indispensable de faire en sorte que le débiteur d'aliments ne puisse se soustraire au devoir aussi impérieux que la loi lui impose. Les sanctions qui garantissent son exécution sont donc plus rigoureuses que celles qui assortissent les obligations ordinaires ». 
Parfois, le débiteur est privé de la possibilité de recourir à des textes à portée générale.

Alors que, d'ordinaire, un débiteur peut résister à une demande en paiement en se prévalant de la compensation de sa propre dette avec une créance dont il est

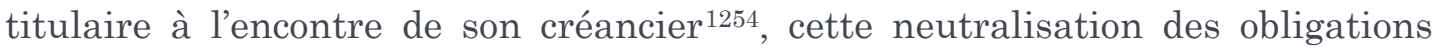
réciproques ne trouve en principe pas application concernant les aliments ${ }^{1255}$.

De même, le délai de grâce, réformé en 1936 et, à cette occasion, stigmatisé comme une des manifestations du "droit de ne pas payer ses dettes" par le Doyen Ripert ${ }^{1256}$, est exclu en matière alimentaire. Alors que, en vertu de l'alinéa $1^{\text {er }}$ de l'article 1244-1 du Code civil, "compte tenu de la situation du débiteur et en considération des besoins du créancier, le juge peut, dans la limite de deux années, reporter ou échelonner le paiement des sommes dues ", l'alinéa $4 \mathrm{du}$ même texte exclut du domaine d'application de ce pouvoir du juge les "dettes d'aliments". La précision n'a, à la réflexion, rien de particulièrement étonnant. On peut en effet la lire comme une présomption irréfragable que la situation de besoin du créancier, que l'article 1244-1 impose de prendre en considération, implique nécessairement le paiement de la créance en temps et en heure ${ }^{1257}$. Il n'en demeure pas moins que faveur va alors, par principe, au

1254 C. civ., art. 1289.

1255 C. civ., art. 1293 : «La compensation a lieu, quelles que soient les causes de l'une ou l'autre des dettes, excepté dans le cas: [...] $3^{\circ}$ D’une dette qui a pour cause des aliments déclarés insaisissables ". Eu égard à la précision portée au texte, des cas exceptionnels de compensation sont envisageables. L'article 14 de la loi $\mathrm{n}^{\circ} 91-650$ du 9 juillet 1991, portant réforme des procédures civiles d'exécution (JO 14 juillet 1991, p. 9228), déclare en effet insaisissables «les provisions, sommes et pensions à caractère alimentaire, sauf pour le paiement des aliments déjà fournis par le saisissant à la partie saisie ». En d'autres termes, celui qui a trop versé au titre de son obligation alimentaire peut saisir les aliments de celui qui s'avère rétrospectivement ne pas être, ou pas à ce point, son créancier d'aliments ("cette exception vise celui qui fournit des aliments en étant trompé sur les réels besoins du créancier et qui va en solliciter la restitution ": Anne LEBORGNE, Voies d'exécution et procédures de distribution, Dalloz, coll. "Précis ", 2009, $n^{\circ} 619$, p. 250). En conséquence, et suivant la même logique, la compensation peut avoir lieu "lorsque la créance réciproque de la créance alimentaire a pour cause une fourniture d'aliments [...] : par exemple, si le débiteur de l'obligation alimentaire a prêté au créancier une somme d'argent pour lui permettre d'acquérir un logement, il peut invoquer la compensation pour éteindre sa dette d'aliments à hauteur de sa créance de restitution de la somme prêtée, qui est alors éteinte simultanément. Dans de semblables hypothèses, le lien obligatoire d'aliments est utilisé conformément à son affectation strictement alimentaire, ce qui justifie pleinement l'exception au principe de non-compensation" (J. Cl. Civil, art. 205 à 211, fasc. 20, précit., par Laurent LEVENEUR, $\mathrm{n}^{\circ} 43$ ).

1256 Georges RIPERT, "Le droit de ne pas payer ses dettes », DH 1936, p. 57, et spéc. p. 60 : "La faveur que la démocratie n'a jamais cessé de témoigner aux faibles lui fait depuis longtemps considérer le débiteur comme le seul digne de la protection des lois ». Il n'en va certes pas de même du débiteur d'aliments ...

1257 Et la Cour de cassation a retenu une interprétation assez large de cette disposition pourtant formellement exceptionnelle: v. Cass. soc. 18 novembre 1992, Bull. civ. V n 555 ; RTD civ. 1993.611, obs. Pierre-Yves GAUTIER. Eu égard à la bonne foi et à la situation difficile de l'employeur, le conseil de prud'hommes l'avait autorisé à s'acquitter de sa dette en huit trimestres. L'arrêt est cassé. Concernant certaines sommes - salaires, primes de fin d'année et indemnité de congés payés -, l'échelonnement est exclu: "s'agissant de créances salariales, le juge ne peut accorder aucun délai de paiement». Alors que la Cour de cassation aurait pu comprendre l'expression " dettes d'aliments » comme un simple renvoi implicite aux articles 205 et suivants du Code civil, elle en adopte une définition assez large - signe d'une certaine rigueur à l'égard de ceux qui doivent fournir aux autres de quoi vivre. La définition des dettes d'aliments n'est toutefois pas 
créancier - faveur au créancier d'aliments qu'exprime aussi, et à contre-courant, le droit du surendettement. En effet, si la saisine de la commission de surendettement des particuliers peut emporter suspension des poursuites, cette solution est exclue en matière de dettes d'aliments ${ }^{1258}$ - qui ne sont pas non plus susceptibles de rééchelonnement au titre de l'article L. 331-7-1 du code de la consommation. De même, le créancier d'aliments jouit d'une situation originale et de faveur - si s'ouvre contre son débiteur une procédure collective régie par le code de commerce ${ }^{1259}$.

Et, si la mise en œuvre des procédures d'exécution forcée s'avère nécessaire, la rigueur à l'égard du débiteur se révèle à nouveau. Tandis que, en principe, certains biens du débiteur sont insaisissables, et en particulier une partie de la rémunération de son travail ${ }^{1260}$, cette insaisissabilité de principe ne pourra pas être opposée au créancier d'aliments, du moins concernant la partie de la rémunération supérieure au seuil du revenu de solidarité active ${ }^{1261}$.

Parfois, des textes spéciaux accroissent la contrainte qui pèse sur le débiteur.

Le créancier d'aliments peut se prévaloir contre le débiteur d'un régime de saisie tout à fait particulier : la procédure de paiement direct instaurée par une loi de $1973^{1262}$. Les conditions posées par les deux premiers alinéas de l'article $1^{\text {er }}$ sont légères : "Tout créancier d'une pension alimentaire peut se faire payer directement le montant de cette pension par les tiers débiteurs de sommes liquides et exigibles envers le débiteur de la pension. Il peut notamment exercer ce droit entre les mains de tout débiteur de salaires, produits du travail ou autres revenus, ainsi que de tout dépositaire de fonds. La demande en paiement direct sera recevable dès qu'une échéance d'une pension alimentaire, fixée par une décision judiciaire devenue exécutoire, n'aura pas été payée à son terme ". L’attrait de la procédure pour le créancier est souligné à l'article 2 selon lequel

indéfiniment extensive. Dans la même décision, l'échelonnement sur deux années du paiement de l'indemnité de licenciement est, lui, admis par la Cour - en raison de son caractère non pas alimentaire mais indemnitaire. Pour une critique, v. Pierre-Yves GAUTIER, obs. précit., p. 612, favorable à une interprétation plus large encore du champ d'application de l'exception au délai de grâce de l'article 1244-1: "au-delà des qualifications savantes, l'on se demandera simplement quelle est la destination de cette indemnité à laquelle a droit un homme ou une femme qui va se retrouver au chômage, pour une durée indéterminée, peut-être très longue, c'est-à-dire dans une des situations les plus douloureuses de la vie contemporaine: la réponse n'est pas difficile, elle l'aidera à assurer sa subsistance et celle de sa famille [...]. En d'autres termes, à supposer qu'une telle indemnité ne soit pas un salaire (cause et nature différentes), elle n'en reste pas moins alimentaire et le délai de grâce devrait de ce fait être normalement exclu ".

1258 C. cons., art. L. 331-5.

1259 Sur ce régime, v. en dernier lieu, Pierre CAGNOLI, "L’obligation alimentaire est-elle une dette spécifique au regard du droit des procédures collectives d'entreprise ? ", LPA 24 juin 2010, p. 37 et s. : "Ici également, la réponse est évidemment affirmative » pose, d'emblée, l'auteur.

1260 C. trav., art. L. $3252-2$, al. $1^{\mathrm{er}}$.

${ }^{1261}$ C. trav., art. L. 3252-5 et R. 3253-5 qui renvoie à l'article L. 262-3 du Code de l'action sociale et des familles - relatif au revenu de solidarité active. Sur la protection du créancier d'aliments ainsi instaurée, v. Anne LEBORGNE, précit., n 1302 et s., p. 498 et s.

${ }_{1262}$ Loi $\mathrm{n}^{\circ} 73-5 \mathrm{du} 2$ janvier 1973 relative au paiement direct de la pension alimentaire, JO 3 janvier 1973, p. 135. 
"la demande vaut, sans autre procédure et par préférence à tous autres créanciers, attribution au bénéficiaire des sommes qui en font l'objet au fur et à mesure qu'elles deviennent exigibles. Le tiers est tenu de verser directement ces sommes au bénéficiaire selon les échéances fixées par le jugement ». Le texte fait donc du créancier d'aliments un créancier privilégié stricto sensu: il bénéficie d'un droit de préférence sur les sommes en question, il sera prioritaire sur tout autre créancier ${ }^{1263}$.

Et la contrainte est d'autant plus forte que le débiteur récalcitrant s'expose à des sanctions pénales spécifiques. "Le fait, pour une personne, de ne pas exécuter une décision judiciaire ou une convention judiciairement homologuée lui imposant de verser au profit d'un enfant mineur, d'un descendant, d'un ascendant ou du conjoint une pension, une contribution, des subsides ou des prestations de toute nature dues en raison de l'une des obligations familiales prévues par le titre IX du livre I ${ }^{\text {er }}$ du code civil, en demeurant plus de deux mois sans s'acquitter intégralement de cette obligation, est puni de deux ans d'emprisonnement et de 15000 euros d'amende "1264. "C'est une des hypothèses remarquables où l'inexécution d'une obligation civile est sanctionnée comme délit pénal »1265. Le délit d'abandon de famille illustre les garanties exceptionnelles dont bénéficie le créancier alimentaire et, réciproquement, l'extrême rigueur du système juridique à l'encontre du débiteur - d'autant que deux autres incriminations peuvent être sollicitées à son encontre ${ }^{1266}$. On constate alors que tout, dans le régime spécifique de l'obligation alimentaire, ne peut être exporté pour garantir la justiciabilité des autres droits sociaux. En effet, les sanctions pénales qui viennent accroître la contrainte qui pèse sur le débiteur s'expliquent par le fait même de l'existence de liens de famille entre les différents acteurs : l'abandon financier ne semble alors que refléter un abandon moral, que l'on peut aisément concevoir comme répréhensible au sein du groupe familial - mais plus difficilement de manière générale.

La justiciabilité de l'obligation alimentaire passe très clairement par un jugement de valeur d'autant plus frappant qu'il ne s'inscrit pas dans la tendance générale du droit contemporain à une relative indulgence pour le débiteur impécunieux : le débiteur doit payer. Cela s'explique : par hypothèse, il n'est pas - ou n'a pas toujours été - sans ressources, sans quoi il ne serait pas débiteur de l'obligation alimentaire, celle-ci étant fonction des besoins du créancier et des ressources du débiteur. Il n'est toutefois pas à l'abri d'une mauvaise gestion

1263 V. Marie-Josèphe GEBLER, «Le paiement direct des pensions alimentaires (Commentaire de la loi n 73-5 du 2 janvier 1973) », D. 1973 Chron 107, spéc. p. 111 : « Le paiement direct n'est pas seulement une voie d'exécution efficace. Il constitue aussi un privilège expressément formulé ". Pour une description précise de la situation du créancier, v. Frédérique EUDIER, "Le recouvrement forcé des créances d'aliments ", LPA 24 juin 2010, p. 41 et s., p. 43 et s.

1264 C. pén., art. 227-3.

1265 Jean CARBONNIER, précit., p. 57.

1266 C. pén., art. 227-4, qui sanctionne le fait, pour un débiteur d'aliments, de n'avoir pas notifié son changement de domicile à son créancier dans un délai d'un mois ; art. 351-3, qui sanctionne l'organisation de son insolvabilité en vue de se soustraire à l'exécution d'une décision de justice, portant en particulier condamnation au paiement d'aliments. 
passagère: elle lui serait pardonnée en toute autre hypothèse et apparaît inadmissible si elle nuit à un créancier d'aliments - sauf à ce que le débiteur obtienne du juge la révision à la baisse, voire la disparition, de sa dette en raison même de l'amenuisement de ses ressources ${ }^{1267}$. Hors ce cas, la garantie des droits de l'un passe par une certaine intransigeance et donc une certaine indifférence à la situation de l'autre. On touche alors certainement à une difficulté de la justiciabilité des droits sociaux : pour que l'un soit rempli de ses droits, il faut demeurer sourd aux intérêts de l'autre. Tous les contextes ne justifient pas, ou pas évidemment, une telle prise de parti : il y a probablement dans la nécessité d'un jugement de valeur très radical de ce type, un frein politique à la justiciabilité des droits sociaux.

\section{B. La mobilisation de l'administration}

En dehors même de l'intervention de l'administration sur le fondement de l'article L. 132-7 du Code de l'action et des familles, qui précède la fixation de la créance $^{1268}$ ou de la question du recours contributif des personnes publiques contre les débiteurs d'aliments, qui n'assure pas stricto sensu un meilleur recouvrement de la créance alimentaire au sens où, alors, le créancier a d'ores et déjà été satisfait, il faut signaler des mécanismes originaux de collaboration de l'administration avec le débiteur ${ }^{1269}$.

En premier lieu, cette collaboration a pour objet la réunion d'informations relatives au débiteur. Ainsi, l'article L. 111-II du Livre des procédures fiscales fait bénéficier les créanciers d'aliments en possession d'un titre exécutoire d'une certaine transparence fiscale: le texte les autorise à accéder à diverses informations parmi lesquelles le revenu imposable du débiteur ${ }^{1270}$. Si la réciproque est vraie, il ne fait pas de doute que cette information fiscale a avant tout pour objet et pour effet de permettre au créancier de se prévaloir de la véritable situation financière du débiteur - c'est-à-dire de prouver que les ressources qui conditionnent l'existence de la créance alimentaire existent bel et bien. Mais des informations plus nombreuses, et plus nombreuses qu'en droit commun, peuvent être réunies par la personne en charge du recouvrement.

\footnotetext{
1267 Sur le fait qu'une telle révision est toujours possible, v. supra p. 418 et 420.

1268 Supra, p. 419.

${ }^{1269}$ Les organismes débiteurs de prestations familiales, personnes privées en charge d'une mission de service public, peuvent aussi être sollicités, mais à une fin particulière : le recouvrement des pensions dont le bénéficiaire est un enfant mineur (Code de la sécurité sociale, art. L 581-1 et s.). D'une part, ils peuvent verser au parent qui ne parvient pas à recouvrer la créance une avance, qui prend la forme de l'allocation de soutien familial (art. L. 581-2). D'autre part, ils peuvent recouvrer la créance au nom et pour le compte du parent en question en vertu d'un mandat irréfragablement présumé du fait même du versement de l'allocation de soutien familial (L. 581-3) ou exprès si le parent n'a pas pu bénéficier de l'allocation en question (art. L. 581-6).

1270 En vertu de l'article L. 111-II du Livre des procédures fiscales, relatif à la publicité de l'impôt : "Les créanciers et débiteurs d'aliments dont la qualité est reconnue par une décision de justice peuvent consulter les éléments des listes mentionnées au I afférents à l'imposition de leur débiteur ou créancier, selon le cas, quelle que soit la direction départementale des finances publiques dans le ressort de laquelle l'imposition du débiteur ou du créancier est établie ". En particulier peut être de la sorte connu le revenu imposable de la personne concernée.
} 
Ainsi, dans le cadre de la procédure de paiement direct ${ }^{1271}$, est-il prévu que, sous certaines réserves, "les administrations au service de l'Etat et des collectivités publiques, les organismes de sécurité sociale et les organismes qui assurent la gestion de prestations sociales sont tenus de réunir et de communiquer, en faisant toutes les diligences nécessaires, à l'huissier de justice chargé par le créancier de former la demande de paiement direct, tous renseignements dont ils disposent ou peuvent disposer permettant de déterminer l'adresse du débiteur de la pension alimentaire, l'identité et l'adresse de son employeur ou de tout tiers débiteur ou dépositaire de sommes liquides ou exigibles ${ }^{1272}$ - sans que l'huissier ait à requérir l'assistance du procureur de la République ${ }^{1273}$. Une disposition équivalente, et qui étend encore le champ des informations accessibles, existe en matière de recouvrement public des pensions alimentaires ${ }^{1274}$.

En second lieu et surtout, le recouvrement peut être assuré directement par l'administration fiscale ${ }^{1275}$ - ce qui évite au créancier un face-à-face que les circonstances familiales peuvent rendre délicat avec le débiteur d'aliments ${ }^{1276}$. Une loi de 1975, relative au recouvrement public des pensions alimentaires ${ }^{1277}$, a en effet instauré un mécanisme original. Dans son dernier état ${ }^{1278}$, l'article $1^{\text {er }}$ de la loi dispose: "Toute pension alimentaire fixée par une décision judiciaire devenue exécutoire dont le recouvrement total ou partiel n'a pu être obtenu par une des voies d'exécution de droit privé peut être recouvrée pour le compte du créancier par les comptables publics compétents". En somme, en matière alimentaire, les agents de l'Etat, dont la saisine est au demeurant fort simple ${ }^{1279}$, peuvent faire office d'huissiers de justice ${ }^{1280}-\mathrm{du}$ moins à titre

\footnotetext{
1271 Supra, p. 423.

${ }^{1272}$ Loi no 73-5 du 2 janvier 1973 relative au paiement direct de la pension alimentaire, précit., art. 7. Les organismes débiteurs de prestations familiales en charge du recouvrement d'une créance en vertu des articles L. 581-1 et suivants du Code de la sécurité sociale peuvent également se prévaloir de cet article (v. l'art. L. 581-8).

1273 Comp. avec les procédures de droit commun posées aux articles 39 et 40 de la loi n 91-650 du 9 juillet 1991 portant réforme des procédures civiles d'exécution (précit.).

${ }^{1274}$ Loi n $75-618$ du 11 juillet 1975, précit., art. 8: les mêmes personnes doivent fournir au comptable public les renseignements dont elles disposent ou peuvent disposer " et qui sont utiles à la mise en œuvre de la procédure de recouvrement public »-sans plus de précision.

1275 Sur cette procédure, v. en part. J. Cl. Civil, art. 205 à 211, fasc. 30 : Aliments - Obligation alimentaire - Mise en œuvre, 2002, par Laurent LEVENEUR, n 87 et s. ; Jean CARBONNIER, précit., p. 56 et p. 61; Frédérique GRANET, art. précit., p. 126 et s., qui la qualifie de "particulièrement énergique» (p. 127).

1276 En ce sens, v. Frédérique EUDIER, art. précit., p. 41 : «Dans un certain nombre de cas, du fait de la survivance de liens affectifs ou de rapports de domination entre le créancier et le débiteur, les tentatives de recouvrement risquent d'intervenir dans un contexte passionnel. C'est pourquoi le législateur a estimé que, dans certains cas, le succès de la procédure de recouvrement était subordonné à l'effacement du créancier d'aliments ".

1277 Loi $n^{\circ} 75-618$ du 11 juillet 1975, relative au recouvrement public des pensions alimentaires, JO 12 juillet 1975, p. 7178.

1278 Issu de l'ordonnance 2010-420 du 27 avril 2010, portant adaptation de dispositions résultant de la fusion de la direction générale des impôts et de la direction générale de la comptabilité publique, JO 30 avril 2010, p. 7839.

${ }^{1279}$ En ce sens, v. Frédérique GRANET, art. précit., p. 126.

1280 Alors que, en principe, les huissiers de justice bénéficient d'un monopole en matière de voies d'exécution posé par l'article 18 de la loi n 91-650 du 9 juillet 1991, portant réforme des
} 
subsidiaire puisque le créancier doit établir avoir recouru à "l'une des voies d'exécution de droit privé " "resté[e] infructueu[se] »1281. Les procédures sont celles applicables en matière de contributions directes ${ }^{1282}$ : la justiciabilité de l'obligation alimentaire entre parents et alliés passe par une mobilisation exceptionnelle des services de l'Etat dans un intérêt privé.

Il ne paraît pas raisonnable de prétendre ériger en modèle de justiciabilité des droits sociaux celle de la créance alimentaire due par les parents et alliés tant influe nécessairement sur la question la nature des débiteurs. Mais il serait inopportun, au nom de l'irréductibilité des situations, de négliger les leçons de l'expérience. Pour conférer au droit à des aliments une plus grande effectivité, le législateur et le juge ont pris acte que le besoin qui en conditionne l'existence en fait une créance incomparable qui appelle une justiciabilité selon des modalités spécifiques. Ces modalités de protection pourraient, autant que de raison, servir de base de réflexion pour penser la justiciabilité des autres droits sociaux.

\section{Pour citer cet article}

Marc Pichard, «La justiciabilité de l’obligation alimentaire entre parents et alliés : quels enseignements pour la justiciabilité des droits sociaux ?», La Revue des Droits de l'Homme, juin 2012 http://revdh.files.wordpress.com/2012/04/la-justiciabilitc3a9-de-__obligation-alimentaire-entreparents-et-allic3a9s3.pdf

procédures civiles d'exécution (précit.). Ce monopole est également malmené lorsque les organismes débiteurs de prestations familiales sont autorisés à recouvrer une créance en vertu des articles L. 581-3 ou L. 581-6 du Code de la sécurité sociale (v. supra note 50).

${ }^{1281}$ Loi n 75-618 du 11 juillet 1975, précit., art. 2, al. 2. L'article 13 de la loi dispense le créancier d'un tel préalable s'il a déjà fallu recourir à la procédure de recouvrement public et s'il souffre d'un nouveau retard de paiement de plus d'un mois.

${ }^{1282}$ Loi n $75-618$ du 11 juillet 1975, précit., art. 7. 